\title{
Fast Readout of Scintillating Fiber Arrays Using Position-sensitive Photomultipliers
}

\author{
FAROS COLLABORATION
}

\author{
V. Agoritsas ${ }^{2}$, N. Akchurin ${ }^{3}$, A. M. Bergdolt ${ }^{8}$, A. Bravar ${ }^{3}$, O. Bing ${ }^{8}$, J. Ditta ${ }^{1}$, \\ J. Dufournaud ${ }^{1}$, R. Giacomich ${ }^{9}$, A. M. Gorin ${ }^{7}$, K. Kuroda ${ }^{1}$, D. Magaudda ${ }^{5}$, \\ I.V. Manuilov ${ }^{7}$,A. Michalowicz ${ }^{1}$, C. Newsom ${ }^{3}$, K. Okada ${ }^{4}$, K. Okusawa ${ }^{6}$, \\ Y. Onel ${ }^{3}$, A. Penzo ${ }^{9}$, A.V. Riazantsev7 , V. I. Rykalin ${ }^{7}$, G. Salvato ${ }^{5}$, \\ P. Schiavon ${ }^{9}$, D. Sillou ${ }^{1}$, F. Takeutchi ${ }^{4}$, M. Tareb-Reyes ${ }^{1}$ and T. Yoshida ${ }^{6}$
}

\begin{abstract}
Experimental results obtained with commercially available PSPMs coupled to $0.5 \mathrm{~mm}$-diameter scintillating fiber arrays show space resolution of $<200 \mu \mathrm{m}$ and time resolution better than $1 \mathrm{~ns}$ with a detection efficiency of about $90 \%$. In addition, the preliminary studies indicate the feasibility of separating double-tracks with a minimum distance of $3 \mathrm{~mm}$ using this technology.
\end{abstract}

(To be submitted to Nuclear Instruments and Methods in Physics Research)
1) Annecy, LAPP/IN2P3, France
2) CERN, Switzerland
3) University of Iowa, Iowa, USA
4) Kyoto-Sangyo University, Japan
5) Messina University/INFN, Italy
6) Osaka City University, Japan
7) Serpukhov, IHEP, Russia
8) Strasbourg, CRN/IN2P3, France
9) Trieste University/INFN, Italy 


\section{Introduction}

Scintillating fiber (SciFi) technique is relatively simple, robust, reliable, and cost-effective. It can be implemented for large-area applications with sub-millimeter precision and sub-nanosecond time resolution. In order to fully exploit the merits of SciFi detectors, the readout devices have to match these general properties; in particular the timing characteristics of plastic scintillators $\left(t_{r} \leq 1 \mathrm{~ns}\right)$. This criterion is the guiding principle behind our choice of position-sensitive photomultiplier tubes (PSPM) as readout devices for SciFi arrays. Compared to different types of photosensitive devices, such as optoelectronic systems (image intensifier, followed by a CCD) [1], visiblelight photon-counters (VLPC) [2] and avalanche photodiodes [3], the photomultiplier is continuing to be a major component in many high-energy experiments due to its fast response, high gain, low noise and good linearity. It is also reliable and simple to operate. Furthermore, the recent introduction of fine-mesh dynodes has brought new dimensions to PMT technology thereby making position sensitivity and immunity to high external magnetic fields possible [4].

The main features of the PSPM+SciFi combination can be summarized as follows:

a) simple and reliable operation,

b) high sensitivity and low noise,

c) good space resolution,

d) excellent time resolution,

e) high rate capacity,

f) self-triggering,

g) immunity against magnetic field,

h) high radiation hardness, and

i ) flexible geometry.

Some of these properties, while considered individually, may be in close competition with those of other techniques. When considered as a whole, however, PSPM+SciFi is a unique technique with a multitude of properties in a single device. For example, combined fast response and high resolution of this type of a device allow to perform a unique function (trigger and tracking) which is generally performed with the aid of two separate devices.

In this paper we report on recent progress in the construction and operation of SciFi arrays coupled to a PSPM. These detectors may prove to find use in the high luminosity environment of future collider experiments. The studies reported here are the natural continuation of an earlier study using coarser prototypes with $1 \mathrm{~mm}$ diameter fibers [6]. 


\section{Description of the Prototypes}

We have constructed two hodoscopes. The first one, referred to as Type$\mathrm{A}$, is constructed from $0.5 \mathrm{~mm}$ diameter scintillating fibers (S101-A, OPTECTRON). As illustrated in Figure 1, the SciFi hodoscope is $28 \mathrm{~mm}$ wide and consists of 5 single-layers of fibers assembled by staggering each layer by $0.25 \mathrm{~mm}$. On the readout end, the SciFi array is split into 4 segments of $7 \mathrm{~mm}$ each. Each segment is stretched over $35 \mathrm{~mm}$ on the photocathode in order to provide a magnification factor of $\alpha=5$, and a space resolution of $\sigma \approx 200 \mu \mathrm{m}$.

A commercially available 3-inch diameter PSPM (Hamamatsu R2486) was used for some of the measurements. R2486 is a 12-stage PSPM with $16 x$ and $16 y$-crossed wire anodes.

The associated electronics are shown schematically in Figure 2. All the anodes (of each $x$ or $y$ array) are connected to a common delay-line with an interval of $3 \mathrm{~ns}$. One can thus measure the relative time difference between the PMT anode pulses going out of the two ends of the delay-line. The anode pulses are then amplified by a factor of 100, discriminated by constant fraction discriminator (CFD) and then fed into a TDC and a real time digitizer (RTD). Using the timing information from the $y$ delay-line, one can determine the hit segment. Similarly, from the $x$ delay-line, one gets the position within the segment.

The second hodoscope prototype, referred to as Type-B, shown in Figure 3 , is designed to study the two-track resolution. Two-track events can be artificially generated by a double-array structure providing one of the array movable with respect to the other. Both arrays are seen by the same rows of pad anodes of a R3941 - Mod. PSPM (8 x 29 pad anodes), custom manufactured by Hamamatsu.

\section{Experimental Results}

We have installed the apparatus in a test-beam $(5-10 \mathrm{GeV} / c$ charged particles) of the CERN PS (Figure 4). It consists of 2 beam defining counters $\mathrm{B} 1$ and B2, two narrow finger counters S1 and S2 $(0.5 \mathrm{~mm}$ wide across the beam) and three SciFi-PSPM hodoscopes. Two planes (X-Y) of Type-A hodoscopes are dedicated to study the space and time resolution as well as two-dimensional imaging, while Type-B is used for studying the reconstruction of two-hit events.

\subsection{Space Resolution}

The space resolution of a hodoscope was studied by using two finger counters placed up- and down-stream of the hodoscope. Fig 5 presents event distribution obtained by putting these counters in coincidence with the 
hodoscope. Also shown is a Gaussian distribution providing the best fit with $\sigma=250 \mu \mathrm{m}$. After unfolding the width of the definition finger counters $(0.5$ $\mathrm{mm}$ ) the resolution is estimated to $\sim 200 \mu \mathrm{m}$, as already confirmed by our preceding measurement using a self calibrating method [7].

\subsection{Linearity of the Reconstructed Position}

The linearity of the reconstructed position was measured by scanning the full width of the SciFi array with one of the finger counters in steps of $0.5 \mathrm{~mm}$. The results are shown in Figure 6.

The linearity within each of the 4 segments is good but slight nonlinearities are seen near the boundaries. This effect is due to the edge of a segment being too close to the edge of the PSPM sensitive area. Note that the width of the segments on the photocathode is $35 \mathrm{~mm}$, while the signals are read out with 12 anodes, each separated by $3.75 \mathrm{~mm}$. This configuration provides only one guard anode beyond the SciFi array segment extremities.

\subsection{Detection Efficiency}

The local detection efficiency has been studied by normalizing the number of events detected by the last dynode of the PSPM with respect to the incident flux as defined by the finger counter (last-dynode.B1.B $\mathrm{B}_{2} \cdot \mathrm{S}_{1} / \mathrm{B}_{1} . \mathrm{B}_{2} \cdot \mathrm{S}_{1}$ ). This raw efficiency, not including any losses by the position reconstruction, is plotted in Figure 7 over the full width of the SciFi array. It is greater than $90 \%$ almost everywhere, except at the boundaries of the SciFi segments.

Note that the areas of lower efficiencies correspond to a periphery of the photocathode -- about $1 \mathrm{~cm}$ width around the edge --. Another local effect that was previously observed [6] at each break between adjacent segments of the fiber array on the photocathode has been improved by arranging the segments on the photocathode in alternate readout directions. This guarantees that the adjacent hit fibers on the sensitive area remain adjacent on the PSPM face, even though they belong to different segments.

The present limit of the average efficiency, $\sim 90 \%$, is due mainly to the amount of light produced in the fibers, and the attenuation along the fiber. The optical contact between SciFi and the photocathode was made with silicon grease. In this connection, not only the quality of the scintillating fiber, but the geometry of the array structure also effects the efficiency. For example, the stacking of 5 single layers locally provides only 2 fibers traversed by incident particles. The thickness of the cladding being $\approx 3 \%$ of the fiber diameter ( $\approx 15 \mu \mathrm{m}$ for $0.5 \mathrm{~mm}$ fibers), the portion of such a weak region presents $\approx 6 \%$ of the array surface.

\subsection{Time Characteristics}


Another important issue concerns the time characteristics of the readout system based on the delay-line method. These characteristics were studied by computing the mean-time, $\left(t_{L}+t_{R}\right) / 2$, with respect to a reference time $t_{0}$, where $t_{L}$ and $t_{R}$ are the TDC contents corresponding to the arrival times of the left and right propagating signals coming out of the delay-line, and $t_{0}$ is generated by either the last dynode of the PSPM, or an external counter. Figure 8 shows the time sum distribution with respect to $t_{0}$ as defined by the last dynode signal of the PSPM. The two peaks were obtained by adding a delay of $10 \mathrm{~ns}$ to $\mathrm{t}_{\mathrm{L}}$ in order to provide a calibration of the time scale. The fluctuation of the mean-time $\left(t_{1}+t_{2}\right) / 2 \approx 0.8$ ns can be interpreted mainly as a transit- time spread of the signal across the stage of the last dynode to the corresponding anode. Such a good time resolution will allow a fast strobe coincidence, for the reconstruction of multi-hit events, at the level of the RTD as described in [5].

Figure 9 shows a similar result obtained with $\mathrm{t}_{0}$ defined by an external scintillator whose thikness was $5 \mathrm{~mm}$. Assuming that the time resolution of the external counter is roughly comparable with that of the PSPM the resolving time of the hodoscope itself is estimated to be $\sigma_{\mathfrak{t}} \sim 0.8 \mathrm{~ns}$.

\subsection{Two-dimensional Imaging}

The spatial resolution as achieved with this prototype allows one to obtain two dimensional images; as an example, in Figure 10, is shown the profile of a scintillator shaped as the letters "LHC" extending over an area of $\approx$ $8 \times 20 \mathrm{~mm}^{2}$, placed in the beam just downstream the hodoscopes. This profile was obtained in real time from 2 crossed planes of SciFi hodoscopes gated by the shaped scintillator.

\section{Two-track Studies}

A study of the two-track resolution of our detector was made using the Type-B hodoscope shown in Figure 3. This hodoscope consists of two layers of fibers placed transverse to the beam direction. The fibers were interleaved at the face of the PSPM in such a way that a single track through the two layers would illuminate a single spot on the photocathode. When one of the layers was displaced in the direction transverse to the beam, two spots would be illuminated, with their separation determined by the offset between the two layers. In this manner, it was possible to simulate two track signals in a dynamic way. The spacing between the tracks was varied from -5 to $5 \mathrm{~mm}$. In order to easily view the two track outputs of the PSPM, two constant fraction discriminators were connected to the delay-line as shown in Figure 11. The output of these discriminators, as well as the anode pulses from the delay-line are shown for two track events in the photographs in figure 12. The tracks shown are typical examples of two track events produced by 10 
$\mathrm{GeV} / \mathrm{c}$ beam particles passing through the hodoscope. Changing the separation (d) between the two hits from 0 to $5 \mathrm{~mm}$, the discriminator correctly triggers down to about $3 \mathrm{~mm}$. It should be noted that the effective propagation speed of the signal on the delay line is reduced by 20 to $40 \%$ due to the connection of the delay line to the anodes with finite length wires. The present study, although it still remains in a sampling procedure, confirms the possibility of reconstructing two-hits in real time [5].

\section{Discussion and Perspectives}

All the data presented above were obtained under fairly critical conditions on the number of photoelectrons emitted from the photocathode of the PSPM. The thresholds of the CFDs corresponded to approximately 4 photoelectrons.

Recently, a significant improvement was achieved with a SciFi array sample assembled by the Nagoya University group [8]. With 5 layers of 0.5 mm SciFi (SCSF38, KURARAY) about 15 - 20 photoelectrons are seen for minimum ionizing particles, which provide a clear spectrum (Figure 13) well separated from the PSPM noise. This performance allows us to foresee not only a higher detection efficiency but also a better space resolution for the next generation of prototypes in the near future.

Within the framework of RD-17, the PSPM is also being improved by using finer grid dynodes with grid-bars of $200 \mu \mathrm{m}$ wide instead of $400 \mu \mathrm{m}$ for those used in commercial tubes. The lateral electron-spread $\left(\mathrm{w}_{\mathrm{O}}\right)$ at the anode is expected to be $3 \mathrm{~mm}$ FWHM in an axial magnetic field of approximately 500 gauss. Such an upgrade of the PSPM is particularly important for improving the time characteristics as well as the two-hit resolution of SciFi hodoscopes. Digitization of hit positions in real time [6] was performed in the present study only in the determination of the hit segment. In the future studies however we intend to carry out a systematic study and development of a real time digitizer (RTD) to meet not only high rate performance demands but also that of good reconstruction of multi-hit events in real time.

\section{Conclusion}

With a spatial resolution better than $200 \mu \mathrm{m}$, time resolution better than $1 \mathrm{~ns}$, two-hit separation of $3 \mathrm{~mm}$, good linearity and efficiency better than $90 \%$, this type of detector is an attractive candidate for high rate topological triggering and tracking applications in particle physics and real time imaging. Relatively simple construction techniques and moderate cost per anode channel are encouraging in view of the needs of future large scale systems. In addition, in view of forthcoming developments in the field of upgraded PSPMs, there is ample room for further improvements. Future efforts will be dedicated to improving the quality of the SciFi arrays as well as the time characteristics of the front-end electronics. 


\section{Acknowledgments}

We are very grateful to the members of the CERN PS group, who offered us the beam facilities under the best conditions. We thank the members of Nagoya University group for the SciFi array samples as well as for their kind communications on the SciFi characteristics and the array assembling. We are much indebted to the technical personnel in the collaborating institutions, in particular, H. Vey, and S. Rico of LAPP as well as S. Reia and D. Iugovaz of INFN/Trieste.

\section{References}

[1] Among several papers in this field, see e.g., R.E. Ansorge et al., Nuclear Instruments and Methods A265 (1988) 33.

[2] M. Atac and M. D. Petroff, Proc. Symp. on Detector R\&D for the SSC, Fort Worth, Texas, 1990.

[3] W. Bruckner et al., Nuclear Instruments and Methods A313 (1992) 429.

[4] K. Kuroda, D. Sillou and F. Takeutchi, Rev. Sci. Instr. 52 (1981) 337.

[5] V. Agoritsus et al., CERN/DRDC 91- 8, DRDC/P-25.

[6] K. Kuroda, F. Lehar, S. Makino, A. Michalowicz, A. Penzo and F. Takeutchi, Nuclear Instruments and Methods A300 (1991) 259.

[7] A.M.Gorin et al., Nucl. Instr. and Methods A344 (1994) 220.

[8] T. Nakano et al., DPNU-91-53, Nov/91; Proceeding of the IEEE 1991, Nuclear Science Symposium, November 5 -9, 1991, Santa Fe, New Mexico.

\section{Figure captions}


Figure 1 Mechanical structure of the SciFi array coupled to the PSPM (R2486).

Figure 2 Block diagram of the readout electronics showing a) the PMT $x$-anode connections, and b) the real time digitization of hit segments.

Figure 3 "Type-B" hodoscope showing the two movable layers for the simulation of two simultaneous hits.

Figure 4 Experimental layout of the T7-S area at CERN.

Figure 5 Space resolution before unfolding the width of the definition counters $(0.5 \mathrm{~mm})$.

Figure 6 The linearity of the reconstructed position is shown as a function of track position, as determined by a movable defining counter. Vertical lines indicate the boundaries between adjacent segments.

Figure 7 Raw detection efficiency versus position. Events were triggered using the last dynode signals of the PSPM. The output discriminators of the delay-line were tested using both leading edge and constant fraction discriminators.

Figure 8 An internal mean-time $\left(t_{L}+t R\right) / 2$ distribution obtained with the reference time $t_{0}$ defined by the last dynode. The two peaks were obtained by shifting the tL signal by 10 nsrered ddddddr e derf.

Figure 9 A mean-time $\left(t_{L}+t_{R}\right) / 2$ distribution obtained with the reference time $t_{0}$ defined by an external counter.

Figure 10 An example of two-dimensional images obtained in real time with 2 planes of hodoscopes. The letters extend over an area $\approx 8 \times 20 \mathrm{~mm}^{2}$

Figure 11 Separation of two simultaneous hits.

Figure 12 Typical example of two-hit events with different "track" separation.

Figure 13 Pulse shape of the PSPM observed with the Nagoya SciFi array showing a) Anode signal through the delay-line and b) the last dynode signal. 
$\begin{array}{llllllll}\text { S1 } & \text { S2 } & \text { S3 } & \text { S4 } & \text { S1 } & \text { S2 } & \text { S3 } & \text { S4 }\end{array}$

$B E A M$

One Segment of

SciFi Hodoscope

On Photocathode

Surface

Aspect Ration 5

PSPM (R2486)

$35 \mathrm{~mm} \quad 30 \mathrm{~mm} \quad 38.1 \mathrm{~mm} \quad 3.75 \mathrm{~mm} \quad 28 \mathrm{~mm}$

Y X

Position of Definition Counter (mm)

Reconstructed Position ( $0.1 \mathrm{~mm} / \mathrm{div}$.)

Reconstructed Position ( $0.1 \mathrm{~mm} / \mathrm{div}$.)

Number of Events

Gaussian Distribution

with $\sigma=250 \mu \mathrm{m}$

Publication

i $n$ NIM

\# 1 : 12 July 1994 


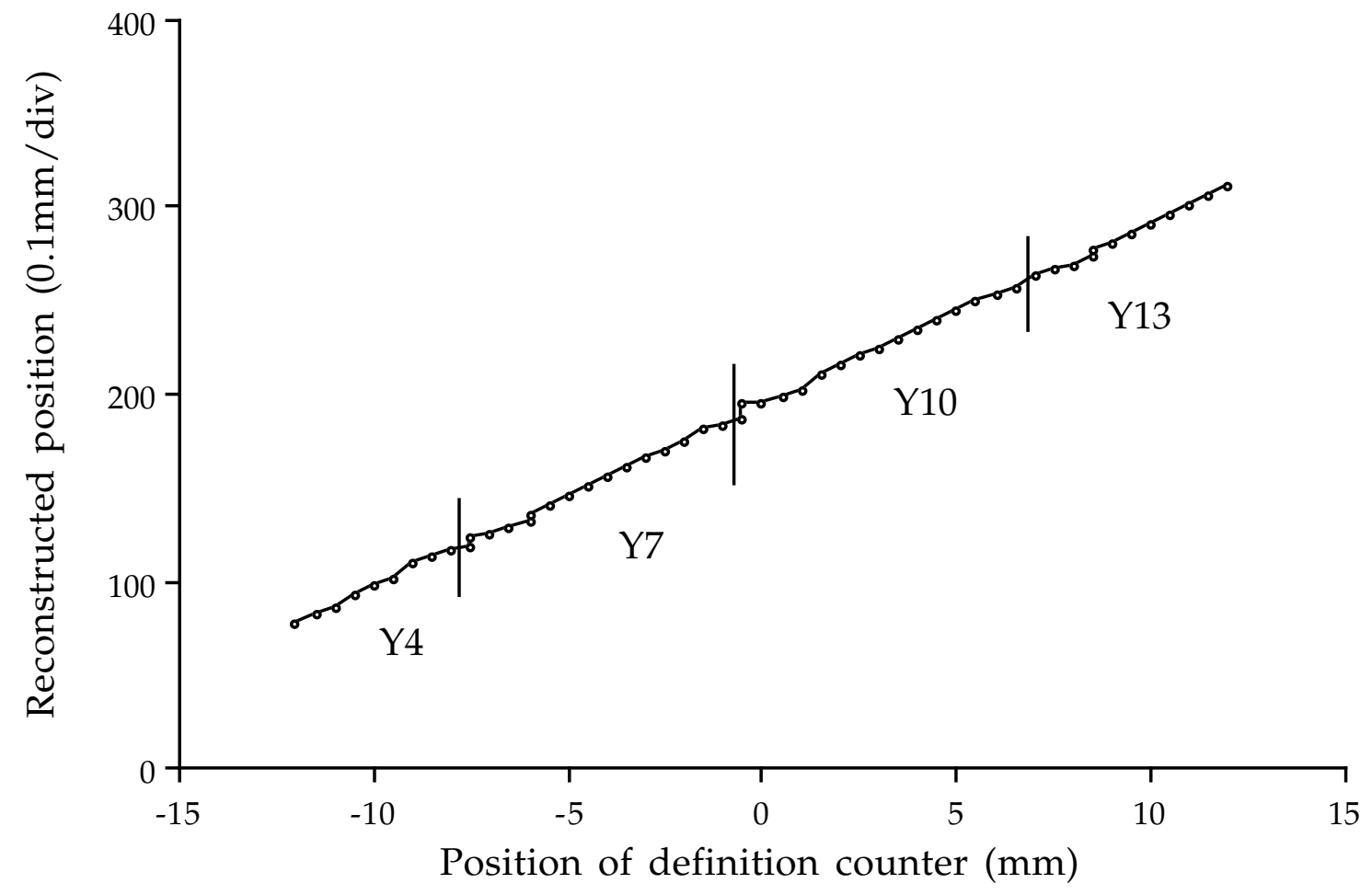




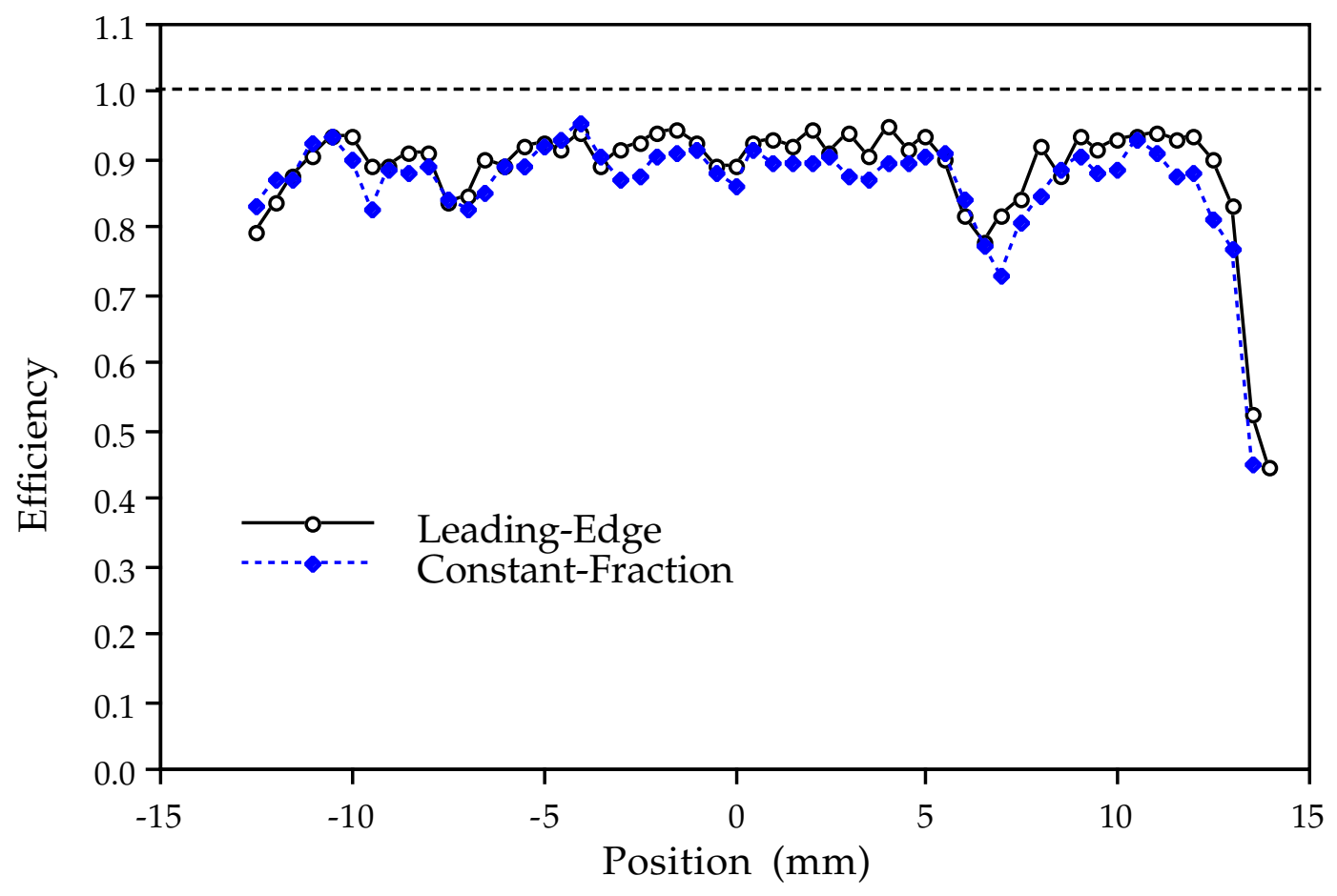

